\title{
The nature of the essential spectrum in curved quantum waveguides *
}

\author{
David Krejčiř́ík $^{1 \dagger}$ and Rafael Tiedra de Aldecoa ${ }^{2}$ \\ 1 Departamento de Matemática, Instituto Superior Técnico, \\ Av. Rovisco Pais, 1049-001 Lisboa, Portugal \\ 2 Département de Physique Théorique, Université de Genève, \\ 24, quai E. Ansermet, 1211 Genève 4, Switzerland \\ E-mail:dkrej@math.ist.utl.pt and rafael.tiedra@physics.unige.ch
}

28 March 2004

\begin{abstract}
We study the nature of the essential spectrum of the Dirichlet Laplacian in tubes about infinite curves embedded in Euclidean spaces. Under suitable assumptions about the decay of curvatures at infinity, we prove the absence of singular continuous spectrum and state properties of possible embedded eigenvalues. The argument is based on Mourre conjugate operator method developed for acoustic multistratified domains by Benbernou in [3] and Dermenjian et al. in [10]. As a technical preliminary, we carry out a spectral analysis for Schrödinger-type operators in straight Dirichlet tubes. We also apply the result to the strips embedded in abstract surfaces.
\end{abstract}

\footnotetext{
*Published in J. Phys. A 37 (2004), no. 20, pp. 5449-5466.

${ }^{\dagger}$ On leave of absence from Department of Theoretical Physics, Nuclear Physics Institute, Academy of Sciences, 25068 Režn near Prague, Czech Republic.
} 


\section{Introduction}

A strong physical motivation to study the Dirichlet Laplacian in infinitely stretched tubular regions comes from the fact it constitutes a reasonable model for the Hamiltonian of a non-relativistic quantum particle in mesoscopic systems called quantum waveguides [11, 26. 19. Since there exists a close relation between spectral and scattering properties of Hamiltonians, one is naturally interested in carrying out the spectral analysis of the Laplacian in order to understand the quantum dynamics in waveguides. For instance, the crucial step in most proofs of asymptotic completeness is to show that the Hamiltonian has no singular continuous spectrum [28]. The Laplacian in a tube has attracted considerable attention since it was shown in [15] that there may be discrete eigenvalues in curved waveguides. However, a detailed analysis of the essential part of the spectrum has been left aside up to now. The purpose of the present paper is to fill in this gap.

The usual model for a curved quantum waveguide, which we adopt in this paper, is as follows. Let $s \mapsto p(s)$ be an infinite unit-speed smooth curve in $\mathbb{R}^{d}, d \geq 2$ (the physical cases corresponding to $d=2,3$ ). Assuming that the curve possesses an appropriate smooth Frenet frame $\left\{e_{1}, \ldots, e_{d}\right\}$ (cf. Assumption 3.1), the $i^{\text {th }}$ curvature $\kappa_{i}$ of $p$, $i \in\{1, \ldots, d-1\}$, is a smooth function of the arc-length parameter $s \in \mathbb{R}$. Given a bounded open connected set $\omega$ in $\mathbb{R}^{d-1}$ with the centre of mass at the origin, we identify the configuration space $\Gamma$ of the waveguide with a tube of cross-section $\omega$ about $p$, namely:

$$
\Gamma:=\mathscr{L}(\mathbb{R} \times \omega), \quad \mathscr{L}\left(s, u^{2}, \ldots, u^{d}\right):=p(s)+u^{\mu} \mathcal{R}_{\mu}^{\nu}(s) e_{\nu}(s),
$$

where $\mu, \nu$ are summation indices taking values in $\{2, \ldots, d\}$ and $\left(\mathcal{R}_{\mu}^{\nu}\right)$ is a family of rotation matrices in $\mathbb{R}^{d-1}$. In this paper, we choose the rotations in such a way that $(s, u)$, with $u:=\left(u^{2}, \ldots, u^{d}\right)$, are orthogonal "coordinates" (cf. Section 3.1.3) due to the technical simplicity. It should be stressed here that while the shape of the tube $\Gamma$ is not influenced by a special choice of $\left(\mathcal{R}_{\mu}^{\nu}\right)$ provided $\omega$ is circular, this may no longer be true for a general cross-section. We make the hypotheses (Assumption 3.2 that $\kappa_{1}$ is bounded, $a\left\|\kappa_{1}\right\|_{\infty}<1$, with $a:=\sup _{u \in \omega}|u|$, and $\Gamma$ does not overlap itself so that the tube can be globally parameterised by $(s, u)$. Our object of interest is the Dirichlet Laplacian associated with the tube, i.e.,

$$
-\Delta_{\mathrm{D}}^{\Gamma} \quad \text { on } \quad L^{2}(\Gamma) .
$$

If $p$ is a straight line, i.e., all $\kappa_{i}=0$, then $\Gamma$ may be identified with the straight tube $\Omega:=\mathbb{R} \times \omega$. In that case, it is easy to see that the spectrum of (1.2) is purely absolutely continuous and equal to the interval $\left[\nu_{1}, \infty\right)$, where $\nu_{1}$ denotes the first eigenvalue of the Dirichlet Laplacian in the cross-section $\omega$.

On the other hand, if $p$ is non-trivially curved and straight asymptotically, in the sense that the curvature $\kappa_{1}$ vanishes at infinity, then the essential spectrum of (1.2) remains equal to $\left[\nu_{1}, \infty\right)$. However, there are always discrete eigenvalues below $\nu_{1}$. When $d=2$, the latter was proved for the first time in [15] for a rapidly decaying curvature and sufficiently small $a$. Numerous subsequent studies improved and generalised this initial result [17, 30, 11, 23, 24, 8]. The generalisation to tubes of circular cross-section in $\mathbb{R}^{3}$ 
was done in [17] (see also [11]) and the case of any dimension $d \geq 2$ and arbitrary cross-section can be found in [8]. Let us also mention that the discrete spectrum may be generated by other local perturbations of the straight tube $\Omega$ (see, e.g., [7, 16 4]), but in the bent-tube case the phenomenon is of a purely quantum origin because there are no classical closed trajectories, apart from those given by a zero measure set of initial conditions in the phase space.

The main goal of the present work is a thorough analysis of the essential spectrum of (1.2). In particular, we find sufficient conditions which guarantee that the essential spectrum of a curved tube "does not differ too much" from the straight case (for simplicity, we present here our results only for $d=2$, see Theorem 3.5 for the $d$-dimensional case):

Theorem $1.1(\boldsymbol{d}=\mathbf{2})$. Let $\Gamma$ be as above for $d=2\left(\kappa:=\kappa_{1}\right)$ and $\mathcal{T}:=\left\{n^{2} \nu_{1}\right\}_{n=1}^{\infty}$ with $\nu_{1}:=\pi^{2} /(2 a)^{2}$ (the set of eigenvalues of the Dirichlet Laplacian in the 1-dimensional cross-section $\omega)$. Suppose

1. $\kappa(s), \ddot{\kappa}(s) \longrightarrow 0$ as $|s| \rightarrow \infty$,

2. $\exists \vartheta \in(0,1]$ s.t. $\dot{\kappa}(s), \dddot{\kappa}(s)=\mathcal{O}\left(|s|^{-(1+\vartheta)}\right)$.

Then

(i) $\sigma_{\text {ess }}\left(-\Delta_{\mathrm{D}}^{\Gamma}\right)=\left[\nu_{1}, \infty\right)$,

(ii) $\sigma_{\mathrm{sc}}\left(-\Delta_{\mathrm{D}}^{\Gamma}\right)=\varnothing$,

(iii) $\sigma_{\mathrm{p}}\left(-\Delta_{\mathrm{D}}^{\Gamma}\right) \cup \mathcal{T}$ is closed and countable,

(iv) $\sigma_{\mathrm{p}}\left(-\Delta_{\mathrm{D}}^{\Gamma}\right) \backslash \mathcal{T}$ is composed of finitely degenerated eigenvalues which can accumulate at points of $\mathcal{T}$ only.

To prove this theorem (and the general Theorem 3.5, we use the conjugate operator method introduced by [27] E. Mourre and lastly developed by [2] W. Amrein et al. Notice that the set $\mathcal{T}$ plays a role analogous to the set of thresholds in the Mourre theory of $N$-body Schrödinger operators [9].

Actually, the property (i) holds true whenever the first curvature vanishes at infinity, without assuming any decay of the derivatives (they may not even exist), see [24] for $d=2$ and [8] for the general case. Our second result (ii) can be compared only with [13] (see also [12]), where the problem of resonances is investigated for $d=2$. Assuming that there exists $\vartheta \in(0,1]$ such that $\kappa(s), \dot{\kappa}(s)^{2}, \ddot{\kappa}(s)=\mathcal{O}\left(|s|^{-(1+\vartheta)}\right)$, the authors proved the absence of singular continuous spectrum as a consequence of the completeness of wave operators obtained by standard smooth perturbation methods of scattering theory. Notice that our and their results are independent. Indeed, while we need to require a faster decay of $\dot{\kappa}$ and also impose a condition on $\dddot{\kappa}$, our decay assumptions on $\kappa$ and $\ddot{\kappa}$ are on the contrary much weaker. Our other spectral results (iii) and (iv) (and (ii) for $d \geq 3$ ) are new.

The organisation of this paper is as follows. In Section 2 we consider the Schrödinger-type operator

$$
H:=-\partial_{i} G^{i j} \partial_{j}+V \quad \text { on } \quad \mathcal{H}(\Omega):=L^{2}(\Omega),
$$


subject to Dirichlet boundary conditions, $i$ and $j$ being summation indices taking values in $\{1, \ldots, d\}, G \equiv\left(G^{i j}\right)$ a real symmetric matrix-valued measurable function on $\Omega$ and $V$ the multiplication operator by a real-valued measurable function on $\Omega$. We make Assumption 2.1 and Assumption 2.2 stated below. Adapting the approach of [3, 10] to non-zero $V$ and $G$ different from a multiple of the identity, we study the nature of the essential spectrum of the operator $H$. In particular, we prove the absence of singular continuous spectrum and state properties of possible embedded eigenvalues. The result is contained in Theorem 2.16 and is of independent interest. In Section 3, we apply it to the case of curved tubes 1.1. Using the diffeomorphism $\mathscr{L}: \Omega \rightarrow \Gamma$ and a unitary transformation (ideas which go back to [15]), we cast the Laplacian (1.2) into a unitarily equivalent operator of the form 1.3 for which Theorem 2.16 can be used. The obtained spectral results can be found in Theorem 3.5 (the general version of Theorem 1.1 above). Finally, in Section 4 we similarly investigate the essential spectrum of the Dirichlet Laplacian in an infinite strip in an abstract two-dimensional Riemannian manifold of curvature $K$. The general result is contained in Theorem 4.2 while the case of flat strips, i.e., with $K=0$, is summarised in Theorem 4.3 (the latter involves the curved strips in $\mathbb{R}^{2}$ as a special case).

For the conjugate operator method and notation used in Section 2 the reader is referred to [2] and particularly to short well-arranged reviews of the abstract theory in [3 Sec. 2] or [10, Sec. 1]. A more detailed geometric background for Section 3 and Section 4 can be found in [22, 8] and [18, 23], respectively.

We use the standard component notation of tensor analysis throughout the paper. In particular, the repeated indices convention is adopted henceforth, the range of indices being $1, \ldots, d$ for Latin and $2, \ldots, d$ for Greek. The indices are associated in a natural way with the components of $x \in \mathbb{R} \times \omega$. The partial derivative w.r.t. $x^{i}$ is often denoted by a comma with the index $i$. The brackets $(\cdot)$ are used in order to distinguish a matrix from its coefficients. The symbols $\delta_{i j}$ and $\delta^{i j}$ are reserved for the components of the identity matrix 1 .

\section{Schrödinger-type operators in straight tubes}

\subsection{Preliminaries}

Let $\omega$ be an (arbitrary) bounded open connected set in $\mathbb{R}^{d-1}, d \geq 2$, and consider the straight tube $\Omega:=\mathbb{R} \times \omega$. Our object of interest in this section is the operator given formally by 1.3 , subject to Dirichlet boundary conditions. In addition to the basic properties required for the matrix $G$ and function $V$, we make the following assumptions.

\section{Assumption 2.1.}

1. $\exists C_{ \pm} \in(0, \infty)$ s.t. $C_{-} 1 \leq G(x) \leq C_{+} 1$ for a.e. $x \in \Omega$,

2. $\forall i, j \in\{1, \ldots, d\}, \lim _{R \rightarrow \infty} \operatorname{ess}_{x \in(\mathbb{R} \backslash[-R, R]) \times \omega}\left|G^{i j}(x)-\delta^{i j}\right|=0$,

3. $\exists \vartheta_{1} \in(0,1], C \in(0, \infty)$ s.t. $\left(\left|G^{i j}{ }_{1}(x)\right|\right) \leq C\left\langle x^{1}\right\rangle^{-\left(1+\vartheta_{1}\right)} 1$ for a.e. $x \in \Omega$, 
4. $G_{, i}^{1 i} \in L^{\infty}(\Omega)$.

Here $\langle\cdot\rangle:=\left(1+|\cdot|^{2}\right)^{1 / 2}$ and the inequalities must be understood in the sense of matrices.

\section{Assumption 2.2.}

1. $V \in L^{\infty}(\Omega)$,

2. $\lim _{R \rightarrow \infty} \operatorname{ess~sup}_{x \in(\mathbb{R} \backslash[-R, R]) \times \omega}|V(x)|=0$,

3. $\exists \vartheta_{2} \in(0,1], C \in(0, \infty)$ s.t. $\left|V_{, 1}(x)\right| \leq C\left\langle x^{1}\right\rangle^{-\left(1+\vartheta_{2}\right)}$ for a.e. $x \in \Omega$.

Let us fix some notations. We write $\mathcal{H}^{\nu}(\Omega)$ and $\mathcal{H}_{0}^{\nu}(\Omega), \nu \in \mathbb{R}$, for the usual Sobolev spaces [1]. Given two Hilbert spaces $\mathcal{H}_{1}$ and $\mathcal{H}_{2}$, we denote by $\mathscr{B}\left(\mathcal{H}_{1}, \mathcal{H}_{2}\right)$, respectively $\mathcal{K}\left(\mathcal{H}_{1}, \mathcal{H}_{2}\right)$, the set of bounded, respectively compact, operators from $\mathcal{H}_{1}$ to $\mathcal{H}_{2}$. We also define $\mathscr{B}\left(\mathcal{H}_{1}\right):=\mathscr{B}\left(\mathcal{H}_{1}, \mathcal{H}_{1}\right)$ and $\mathcal{K}\left(\mathcal{H}_{1}\right):=\mathcal{K}\left(\mathcal{H}_{1}, \mathcal{H}_{1}\right)$. We denote by $\mathcal{H}_{1}^{*}$ the topological antidual of $\mathcal{H}_{1}$. We write $(\cdot, \cdot)$ for the inner product in $\mathcal{H}(\Omega)$ and $\|\cdot\|$ for the norm in $\mathcal{H}(\Omega)$ and $\mathscr{B}(\mathcal{H}(\Omega))$.

We now give a meaning to the formal expression (1.3). We start by introducing the sesquilinear form $Q_{0}$ on $\mathcal{H}(\Omega)$ defined by

$$
Q_{0}(\varphi, \psi):=\left(\varphi_{, i}, \delta^{i j} \psi_{, j}\right), \quad \varphi, \psi \in \mathcal{D}\left(Q_{0}\right):=\mathcal{H}_{0}^{1}(\Omega),
$$

which is densely defined, symmetric, non-negative and closed. Consequently, there exists a unique self-adjoint operator $H_{0}$ associated with it, which is just the Dirichlet Laplacian $-\Delta_{\mathrm{D}}^{\Omega}$ on $L^{2}(\Omega)$. We have

$$
H_{0} \psi=-\Delta \psi, \quad \psi \in \mathcal{D}\left(H_{0}\right)=\left\{\psi \in \mathcal{H}_{0}^{1}(\Omega): \Delta \psi \in \mathcal{H}(\Omega)\right\} .
$$

We consider $H$ as an operator obtained by perturbing the free Hamiltonian $H_{0}$. Since the matrix $G$ is uniformly positive and bounded by Assumption 2.1 1, the sesquilinear form $(\varphi, \psi) \mapsto\left(\varphi_{, i}, G^{i j} \psi_{, j}\right)$ defined on $\mathcal{D}\left(Q_{0}\right) \times \mathcal{D}\left(Q_{0}\right)$ is also densely defined, symmetric, non-negative and closed. At the same time, the potential $V$ is supposed to be bounded by Assumption 2.2 1, which means that the sesquilinear form $Q$ defined by

$$
Q(\varphi, \psi):=\left(\varphi_{, i}, G^{i j} \psi_{, j}\right)+(\varphi, V \psi), \quad \varphi, \psi \in \mathcal{D}(Q):=\mathcal{H}_{0}^{1}(\Omega),
$$

gives rise to a semi-bounded self-adjoint operator $H$. Using the representation theorem [21, Chap. VI, Thm. 2.1] and the fact that $V$ is bounded (recall also Assumption 2.11), one may check that

$$
\mathcal{D}(H)=\left\{\psi \in \mathcal{H}_{0}^{1}(\Omega): \partial_{i} G^{i j} \partial_{j} \psi \in \mathcal{H}(\Omega)\right\},
$$

where the derivatives must be interpreted in the distributional sense, and that $H$ is acting as in (1.3) on its domain.

For any $z \in \mathbb{C} \backslash \sigma\left(H_{0}\right)$, respectively $z \in \mathbb{C} \backslash \sigma(H)$, let $R_{0}(z):=\left(H_{0}-z\right)^{-1}$, respectively $R(z):=(H-z)^{-1}$. 


\subsection{Localisation of the essential spectrum}

The Dirichlet Laplacian $-\Delta_{\mathrm{D}}^{\omega}$ on $L^{2}(\omega)$, i.e., the operator associated with

$$
q(\varphi, \psi):=\left(\varphi, \mu, \delta^{\mu \nu} \psi_{, \nu}\right), \quad \varphi, \psi \in \mathcal{D}(q):=\mathcal{H}_{0}^{1}(\omega)
$$

has a purely discrete spectrum consisting of eigenvalues $\nu_{1}<\nu_{2} \leq \nu_{3} \leq$.. with $\nu_{1}>0$. We set $\mathcal{T}:=\left\{\nu_{n}\right\}_{n=1}^{\infty}$. Since $H_{0}$ is naturally decoupled in the following way:

$$
H_{0}=-\Delta^{\mathbb{R}} \otimes 1+1 \otimes\left(-\Delta_{\mathrm{D}}^{\omega}\right) \quad \text { on } \quad L^{2}(\mathbb{R}) \otimes L^{2}(\omega),
$$

where " $\otimes$ " denote the closed tensor product, 1 the identity operators on appropriate spaces and $-\Delta^{\mathbb{R}}$ the Laplacian on $L^{2}(\mathbb{R})$, one has

$$
\sigma\left(H_{0}\right)=\sigma_{\text {ess }}\left(H_{0}\right)=\left[\nu_{1}, \infty\right) .
$$

In order to prove that (under our assumptions) $H$ possesses the same essential spectrum, we need the following lemma.

Lemma 2.1. Let $\varphi \in C_{0}^{\infty}(\mathbb{R})$ and set $\phi:=\varphi \otimes 1$ on $\Omega$. Then, as a multiplication operator, $\phi \in \mathcal{K}\left(\mathcal{D}\left(H_{0}\right), \mathcal{H}_{0}^{1}(\Omega)\right)$.

Proof. Since

$$
\phi=H_{0}^{-1 / 2} H_{0}^{1 / 2} \phi H_{0}^{-1} H_{0}
$$

in $\mathscr{B}\left(\mathcal{D}\left(H_{0}\right), \mathcal{H}_{0}^{1}(\Omega)\right), H_{0} \in \mathscr{B}\left(\mathcal{D}\left(H_{0}\right), \mathcal{H}(\Omega)\right)$ and $H_{0}^{-1 / 2} \in \mathscr{B}\left(\mathcal{H}(\Omega), \mathcal{H}_{0}^{1}(\Omega)\right)$, it is enough to prove that $H_{0}^{1 / 2} \phi H_{0}^{-1} \in \mathcal{K}(\mathcal{H}(\Omega))$. However,

$$
\begin{aligned}
H_{0}^{1 / 2} \phi H_{0}^{-1} & =H_{0}^{-1 / 2}\left[H_{0}, \phi\right] H_{0}^{-1}+H_{0}^{-1 / 2} \phi \\
& =-H_{0}^{-1 / 2}\left(2 \phi, 1 \partial_{1}+\phi, 11\right) H_{0}^{-1}+H_{0}^{-1 / 2} \phi
\end{aligned}
$$

where each term on the r.h.s. is in $\mathcal{K}(\mathcal{H}(\Omega))$. Let us demonstrate it for the first term. Since $\partial_{1} H_{0}^{-1} \in \mathscr{B}(\mathcal{H}(\Omega))$, it is sufficient to prove that $H_{0}^{-1 / 2} \phi_{, 1} \in \mathcal{K}(\mathcal{H}(\Omega))$. Let $z_{1} \in$ $(-\infty, 0)$ and $z_{2} \in\left(-\infty, \nu_{1}\right)$ be such that $z_{1}+z_{2}=0$. Define $R_{\Perp}\left(z_{1}\right):=\left(-\Delta^{\mathbb{R}}-z_{1}\right)^{-1}$ and $R_{\perp}\left(z_{2}\right):=\left(-\Delta_{\mathrm{D}}^{\omega}-z_{2}\right)^{-1}$. Then, using some standard results on tensor products of operators [20. Chap. 11], one can write

$$
H_{0}^{-1 / 2} \phi_{, 1}=H_{0}^{-1 / 2}\left[R_{\|}^{-1 / 4}\left(z_{1}\right) \otimes R_{\perp}^{-1 / 4}\left(z_{2}\right)\right]\left[R_{\|}^{1 / 4}\left(z_{1}\right) \varphi, 1 \otimes R_{\perp}^{1 / 4}\left(z_{2}\right)\right]
$$

where $\varphi, 1$ is viewed as a multiplication operator in $L^{2}(\mathbb{R})$. The third factor on the r.h.s. is in $\mathcal{K}(\mathcal{H}(\Omega))$ because $-\Delta_{\mathrm{D}}^{\omega}$ has a compact resolvent and $R_{\|}^{1 / 4}\left(z_{1}\right) \varphi, 1 \in \mathcal{K}\left(L^{2}(\mathbb{R})\right)$ by $[2$, Thm. 4.1.3]. The remaining factors can be rewritten as

$$
\Psi\left(X_{1}, X_{2}\right):=\left(X_{1}+X_{2}\right)^{-1 / 2} X_{1}^{1 / 4} X_{2}^{1 / 4}
$$


with $X_{1}:=\left(-\Delta^{\mathbb{R}}-z_{1}\right) \otimes 1$ and $X_{2}:=1 \otimes\left(-\Delta_{\mathrm{D}}^{\omega}-z_{2}\right)$ (both self-adjoint and mutually commuting). So, one can estimate

$$
\left\|\Psi\left(X_{1}, X_{2}\right)\right\| \leq \sup _{x_{1}, x_{2} \in(0, \infty)}\left(x_{1}+x_{2}\right)^{-1 / 2}\left(x_{1} x_{2}\right)^{1 / 4}<\infty .
$$

Hence, the first term on the r.h.s. of 2.4 is in $\mathcal{K}(\mathcal{H}(\Omega))$. The argument is similar for the remaining terms.

Proposition 2.2. One has

(i) $\forall z \in \mathbb{C} \backslash\left(\sigma(H) \cup \sigma\left(H_{0}\right)\right), R(z)-R_{0}(z) \in \mathcal{K}(\mathcal{H}(\Omega))$,

(ii) $\sigma_{\mathrm{ess}}(H)=\left[\nu_{1}, \infty\right)$.

Proof. We prove (i) for some (and hence for all) value of $z \in \mathbb{C} \backslash\left(\sigma(H) \cup \sigma\left(H_{0}\right)\right)$. Let $z \in \mathbb{C} \backslash \mathbb{R}$. Define $R_{1}(z):=\left(H_{0}+V-z\right)^{-1}$. Then, one has

$$
R(z)-R_{0}(z)=R(z)-R_{1}(z)-R_{1}(z) V R_{0}(z) .
$$

Let us first consider $R(z)-R_{1}(z)$. Knowing that $H$ and $H_{0}+V$ have the same form domain, the identity

$$
R(z)-R_{1}(z)=-R(z)\left(H-H_{0}-V\right) R_{1}(z)
$$

holds in $\mathscr{B}\left(\mathcal{H}^{-1}(\Omega), \mathcal{H}_{0}^{1}(\Omega)\right)$. But, one has the following sequence of continuous and dense imbeddings of Hilbert spaces

$$
\mathcal{D}(H) \subset \mathcal{H}_{0}^{1}(\Omega) \subset \mathcal{H}(\Omega) \subset \mathcal{H}^{-1}(\Omega) \subset \mathcal{D}(H)^{*}
$$

which implies that $R(z)$ extends (by duality) to a homeomorphism of $\mathcal{D}(H)^{*}$ onto $\mathcal{H}(\Omega)$. Thus, since $R_{1}(z)$ is also a homeomorphism from $\mathcal{H}(\Omega)$ onto $\mathcal{D}\left(H_{0}\right), R(z)-R_{1}(z) \in$ $\mathcal{K}(\mathcal{H}(\Omega))$ if and only if $H-H_{0}-V \in \mathcal{K}\left(\mathcal{D}\left(H_{0}\right), \mathcal{D}(H)^{*}\right)$. For all $n \in \mathbb{N} \backslash\{0\}$, let $\varphi_{n} \in C_{0}^{\infty}(\mathbb{R})$ be such that $0 \leq \varphi_{n} \leq 1$ and

$$
\varphi_{n}\left(x^{1}\right)=\left\{\begin{array}{lll}
1 & \text { if } & \left|x^{1}\right| \leq n \\
0 & \text { if } & \left|x^{1}\right| \geq n+1 .
\end{array}\right.
$$

Set $\phi_{n}:=\varphi_{n} \otimes 1$ on $\Omega$ and

$$
K_{n} \psi:=-\partial_{i} F^{i j} \phi_{n} \partial_{j} \psi, \quad \psi \in \mathcal{D}\left(H_{0}\right),
$$

where $\left(F^{i j}\right):=\left(G^{i j}-\delta^{i j}\right)$. Clearly, $H-H_{0}-V, K_{n} \in \mathscr{B}\left(\mathcal{D}\left(H_{0}\right), \mathcal{D}(H)^{*}\right)$ and

$$
\begin{aligned}
& \left\|K_{n}-\left(H-H_{0}-V\right)\right\|_{\mathscr{B}\left(\mathcal{D}\left(H_{0}\right), \mathcal{D}(H)^{*}\right)} \\
& \equiv \sup _{\psi \in \mathcal{D}\left(H_{0}\right),\|\psi\|_{\mathcal{D}\left(H_{0}\right)}=1}\left\|\left(1+H^{2}\right)^{-1 / 2}\left[-\partial_{i} F^{i j}\left(\phi_{n}-1\right) \partial_{j}\right] \psi\right\| \\
& \leq \sup _{\psi \in \mathcal{D}\left(H_{0}\right),\|\psi\|_{\mathcal{D}\left(H_{0}\right)}=1} \sum_{j=1}^{d}\left\|\left(1+H^{2}\right)^{-1 / 2} \partial_{i}\right\|\left\|F^{i j}\left(\phi_{n}-1\right)\right\|_{\infty}\|\psi\|_{\mathcal{H}_{0}^{1}(\Omega)} \underset{n \rightarrow \infty}{\longrightarrow} 0,
\end{aligned}
$$


where we have used the fact that $\mathcal{D}\left(H_{0}\right) \subset \mathcal{H}_{0}^{1}(\Omega)$ continuously and Assumption 2.12 in the final step. So, it only remains to show that $K_{n} \in \mathcal{K}\left(\mathcal{D}\left(H_{0}\right), \mathcal{D}(H)^{*}\right)$. After a commutation, one gets in $\mathscr{B}\left(\mathcal{D}\left(H_{0}\right), \mathcal{D}(H)^{*}\right)$

$$
K_{n}=-\partial_{i} F^{i j} \partial_{j} \phi_{n}+\partial_{i} F^{i 1} \phi_{n, 1}
$$

where $\phi_{n}, \phi_{n, 1}$ are seen as multiplication operators in $\mathcal{H}(\Omega)$. It is clear that both $\partial_{i} F^{i j} \partial_{j}$ and $\partial_{i} F^{i 1}$ are in $\mathscr{B}\left(\mathcal{H}_{0}^{1}(\Omega), \mathcal{D}(H)^{*}\right)$. Moreover, $\phi_{n}$ and $\phi_{n, 1}$ are in $\mathcal{K}\left(\mathcal{D}\left(H_{0}\right), \mathcal{H}_{0}^{1}(\Omega)\right)$ by Lemma 2.1 Thus, $K_{n} \in \mathcal{K}\left(\mathcal{D}\left(H_{0}\right), \mathcal{D}(H)^{*}\right)$ so that $R(z)-R_{1}(z) \in \mathcal{K}(\mathcal{H}(\Omega))$. Using similar arguments, one can also prove that the $R_{1}(z) V \phi_{n} R_{0}(z)$ is compact and converges to $R_{1}(z) V R_{0}(z)$ in $\mathscr{B}(\mathcal{H}(\Omega))$ due to Assumption 2.2 2. This implies that $R_{1}(z) V R_{0}(z) \in \mathcal{K}(\mathcal{H}(\Omega))$.

(ii) It is a direct consequence of (i), (2.3) and Weyl's theorem [29, Thm. XIII.14].

Remark 2.3. Notice that Assumptions 2.1],2.14 and 2.2 3 are not used in the proof of Proposition 2.2

\subsection{Nature of the essential spectrum}

This part is devoted to a more detailed analysis of the essential spectrum of $H$. In particular, we show that the singular continuous spectrum is empty. The strategy adapted from [3] is the following. Firstly, we construct a dilation operator $A$ such that $H_{0} \in C^{\infty}(A)$ and $H \in C^{1+\vartheta}(A)$ with $\vartheta:=\min \left\{\vartheta_{1}, \vartheta_{2}\right\} \in(0,1]$ (see [2], [3] Sec. 2] or [10, Sec. 1] for definitions of the spaces involved here and in the sequel). Secondly, we prove that $A$ is strictly conjugate (in Mourre's sense) to $H_{0}$ on $\mathbb{R} \backslash \mathcal{T}$. Finally, since $R(i)-R_{0}(i)$ is compact by the first claim of Proposition 2.2 and both $H$ and $H_{0}$ are of class $C_{u}^{1}(A) \supseteq$ $C^{1+\vartheta}(A) \supseteq C^{\infty}(A)$, it follows that $A$ is conjugate to $H$ on $\mathbb{R} \backslash \mathcal{T}$ as well.

\subsubsection{The dilation operator}

Let $q^{1}$ be the multiplication operator by the coordinate $x^{1}$ in $\mathcal{H}(\Omega)$. Let

$$
A:=\frac{1}{2}\left(q^{1} p_{1}+p_{1} q^{1}\right) \quad \text { with } \quad p_{1}:=-i \partial_{1}
$$

be the dilation operator in $\mathcal{H}(\Omega)$ w.r.t. $x^{1}$, i.e., the self-adjoint extension of the operator defined by the expression (2.5) with $C_{0}^{\infty}(\Omega)$ as initial domain. Define $A_{\|}$as the selfadjoint operator in $L^{2}(\mathbb{R})$ such that $A=A_{\| 1} \otimes 1$.

Remark 2.4. The group $\left\{\mathrm{e}^{i A t}\right\}_{t \in \mathbb{R}}$ leaves invariant $\mathcal{H}_{0}^{1}(\Omega)$. Indeed, using the natural isomorphism $\mathcal{H}_{0}^{1}(\Omega) \simeq \mathcal{H}^{1}(\mathbb{R}) \otimes \mathcal{H}_{0}^{1}(\omega)$, one can write

$$
\forall t \in \mathbb{R}, \quad \mathrm{e}^{i A t} \mathcal{H}_{0}^{1}(\Omega)=\left(\mathrm{e}^{i A_{\|} t} \mathcal{H}^{1}(\mathbb{R})\right) \otimes \mathcal{H}_{0}^{1}(\omega) .
$$

Then, the affirmation follows from the fact [2, Prop. 4.2.4] that $\mathcal{H}^{1}(\mathbb{R})$ is stable un$\operatorname{der}\left\{\mathrm{e}^{i A_{\|} t}\right\}_{t \in \mathbb{R}}$. 
In order to deal with the commutator $i[H, A]$, we need the following family of operators

$$
\left\{p_{1}(\varepsilon):=p_{1}\left(1+i \varepsilon p_{1}\right)^{-1}\right\}_{\varepsilon>0},
$$

which regularises the momentum operator $p_{1}$ :

Lemma 2.5. One has

(i) $\left\{p_{1}(\varepsilon)\right\}_{\varepsilon>0} \subset \mathscr{B}(\mathcal{H}(\Omega))$,

(ii) $\left\{p_{1}(\varepsilon)\right\}_{\varepsilon>0}$ is uniformly bounded in $\mathscr{B}\left(\mathcal{H}^{1}(\Omega), \mathcal{H}(\Omega)\right)$ and $\mathrm{s}-\lim _{\varepsilon \rightarrow 0} p_{1}(\varepsilon)=p_{1}$ in $\mathscr{B}\left(\mathcal{H}^{1}(\Omega), \mathcal{H}(\Omega)\right)$,

(iii) $\forall \varepsilon>0,\left[p_{1}(\varepsilon), q_{1}\right]=-i\left(1+i \varepsilon p_{1}\right)^{-2}$ in $\mathscr{B}(\mathcal{H}(\Omega))$,

(iv) $\forall \varepsilon>0, p_{1}(\varepsilon) \mathcal{H}_{0}^{1}(\Omega) \subset \mathcal{H}_{0}^{1}(\Omega)$.

Proof. The first three assertions are established in [3] Lemma 4.1]. Consequently, it only remains to prove the last statement. Using the isomorphism mentioned in Remark 2.4 one can write

$$
\forall \varepsilon>0, \quad p_{1}(\varepsilon) \mathcal{H}_{0}^{1}(\Omega)=-i \varepsilon^{-1}\left\{\left[1+i \varepsilon^{-1}\left(p_{1}-i \varepsilon^{-1}\right)^{-1}\right] \mathcal{H}^{1}(\mathbb{R})\right\} \otimes \mathcal{H}_{0}^{1}(\omega),
$$

where $p_{1}$ on the r.h.s. must be viewed as an operator acting in $L^{2}(\mathbb{R})$. With this last relation, it is clear that $\mathcal{H}_{0}^{1}(\Omega)$ is left invariant by the family $\left\{p_{1}(\varepsilon)\right\}_{\varepsilon>0}$.

We also need the following density result for the set $\mathcal{D}(H)_{c}:=\{\psi \in \mathcal{D}(H)$ : $\operatorname{supp}(\psi)$ is compact\}.

Lemma 2.6. One has

(i) $\mathcal{D}(H)_{c}$ is dense in $\mathcal{D}(H)$,

(ii) $\mathcal{D}(H)_{c}$ is dense in $\mathcal{H}_{0}^{1}(\Omega)$.

Proof. (i) We are inspired by [10, Lemma 2.1]. Let $\psi \in \mathcal{D}(H)$. Define $\varphi_{0} \in C_{0}^{\infty}(\mathbb{R})$ such that

$$
\varphi_{0}\left(x^{1}\right)=\left\{\begin{array}{lll}
1 & \text { if } & \left|x^{1}\right| \leq 1 \\
0 & \text { if } & \left|x^{1}\right| \geq 2 .
\end{array}\right.
$$

Let $n \in \mathbb{N}$. Set $\varphi_{n}\left(x^{1}\right):=\varphi_{0}\left(x^{1} /(n+1)\right)$ for $x^{1} \in \mathbb{R}$ and $\phi_{n}:=\varphi_{n} \otimes 1$ on $\Omega$. Then, $\phi_{n} \psi \in \mathcal{H}_{0}^{1}(\Omega), \lim _{n \rightarrow \infty} \phi_{n} \psi=\psi$ in $\mathcal{H}(\Omega)$ and

$$
H \phi_{n} \psi=\phi_{n} H \psi-2 \phi_{n, 1} G^{1 j} \psi_{, j}-\phi_{n, 11} G^{11} \psi-\phi_{, 1} G_{, i}^{1 i} \psi
$$

in the sense of distributions. Using the fact that $\operatorname{supp}\left(\phi_{n}\right)$ is compact, Assumption 2.1 1 and Assumption 2.14, one has $\phi_{n} \psi \in \mathcal{D}(H)_{\mathrm{c}}$. Moreover, as a consequence of (2.7) and the property

$$
\forall k \in \mathbb{N}, \forall x \in \Omega, \quad \partial_{1}^{k} \phi_{n}(x)=(n+1)^{-k} \varphi_{0}^{(k)}\left(x^{1} /(n+1)\right),
$$


one also has $\lim _{n \rightarrow \infty} H \phi_{n} \psi=H \psi$ in $\mathcal{H}(\Omega)$.

(ii) Using point (i) and the fact that $\mathcal{D}(H) \subset \mathcal{H}_{0}^{1}(\Omega)$ continuously and densely, one gets the following embeddings

$$
\mathcal{H}_{0}^{1}(\Omega)={\overline{\overline{\mathcal{D}(H)_{\mathrm{c}}}}}^{\mathcal{D}(H)} \mathcal{H}_{0}^{1}(\Omega) \subseteq{\overline{\overline{\mathcal{D}(H)_{\mathrm{c}}}}}^{\mathcal{H}_{0}^{1}(\Omega)}{ }^{\mathcal{H}_{0}^{1}(\Omega)}=\overline{\mathcal{D}(H)_{\mathrm{c}}} \mathcal{H}_{0}^{1}(\Omega) \subseteq \mathcal{H}_{0}^{1}(\Omega)
$$

which, in particular, imply that $\mathcal{D}(H)_{\mathrm{c}}$ is dense in $\mathcal{H}_{0}^{1}(\Omega)$.

Now, we can compute the commutator $i[H, A]$.

Proposition 2.7. The sesquilinear form $\mathcal{Q}$ on $\mathcal{H}(\Omega)$ defined by

$$
\mathcal{Q}(\varphi, \psi):=i[(H \varphi, A \psi)-(A \varphi, H \psi)], \quad \varphi, \psi \in \mathcal{D}(\mathcal{Q}):=\mathcal{D}(H) \cap \mathcal{D}(A),
$$

is continuous on $\mathcal{D}(H)_{\mathrm{c}}$ for the topology induced by $\mathcal{H}_{0}^{1}(\Omega)$. Moreover,

$$
i[H, A]=-\partial_{j} G^{1 j} \partial_{1}-\partial_{1} G^{1 j} \partial_{j}+\partial_{i} q^{1} G_{, 1}^{i j} \partial_{j}-q^{1} V_{, 1}
$$

as operators in $\mathscr{B}\left(\mathcal{H}_{0}^{1}(\Omega), \mathcal{H}^{-1}(\Omega)\right)$.

Proof. Let $\varphi, \psi \in \mathcal{D}(H)_{c}$. Using the identity $A=q^{1} p_{1}-\frac{i}{2}$ valid on $\mathcal{D}(H)_{c} \subset \mathcal{D}(A)$, we have

$$
\begin{aligned}
\mathcal{Q}(\varphi, \psi)= & i[(H \varphi, A \psi)-(A \varphi, H \psi)] \\
= & (\varphi, H \psi)+i\left[\left(-\partial_{i} G^{i j} \partial_{j} \varphi, q^{1} p_{1} \psi\right)-\left(q^{1} p_{1} \varphi,-\partial_{i} G^{i j} \partial_{j} \psi\right)\right] \\
& +\left(V \varphi, q^{1} \psi, 1\right)+\left(q^{1} \varphi, 1, V \psi\right) .
\end{aligned}
$$

In order to justify the subsequent integration by parts, we employ the family 2.6. Since $\psi$ has a compact support and belongs to $\mathcal{H}_{0}^{1}(\Omega)$, it follows by using properties (iii) and (iv) of Lemma 2.5 that $q^{1} p_{1}(\varepsilon) \psi \in \mathcal{H}_{0}^{1}(\Omega)$ for all $\varepsilon>0$. So, we can write

$$
\begin{aligned}
\left(-\partial_{i} G^{i j} \partial_{j} \varphi, q^{1} p_{1} \psi\right) & =\lim _{\varepsilon \rightarrow 0}\left(-\partial_{i} G^{i j} \partial_{j} \varphi, q^{1} p_{1}(\varepsilon) \psi\right) \\
& =\lim _{\varepsilon \rightarrow 0}\left(\varphi_{, j}, G^{i j} \partial_{i} q^{1} p_{1}(\varepsilon) \psi\right) \\
& =-i\left(\varphi_{, j}, G^{1 j} \psi_{, 1}\right)+\lim _{\varepsilon \rightarrow 0}\left(\varphi_{, i}, G^{i j} q^{1} p_{1}(\varepsilon) \psi_{, j}\right)
\end{aligned}
$$

and similarly for the integral

$$
\left(q^{1} p_{1} \varphi,-\partial_{i} G^{i j} \partial_{j} \psi\right)=i\left(\varphi, 1, G^{1 j} \psi_{, j}\right)+\lim _{\varepsilon \rightarrow 0}\left(p_{1}(\varepsilon)^{*} \varphi_{, i}, q^{1} G^{i j} \psi_{, j}\right) .
$$

Since

$$
\begin{aligned}
\lim _{\varepsilon \rightarrow 0}\left(p_{1}(\varepsilon)^{*} \varphi_{, i}, q^{1} G^{i j} \psi_{, j}\right)= & \lim _{\varepsilon \rightarrow 0}\left(\varphi_{, i}, p_{1}(\varepsilon) q^{1} G^{i j} \psi_{, j}\right) \\
= & -i\left[\left(\varphi_{, i}, G^{i j} \psi_{, j}\right)+\left(\varphi_{, i}, q^{1} G_{, 1}^{i j} \psi_{, j}\right)\right] \\
& +\lim _{\varepsilon \rightarrow 0}\left(\varphi_{, i}, q^{1} G^{i j} p_{1}(\varepsilon) \psi_{, j}\right)
\end{aligned}
$$


and

$$
\left(q^{1} \varphi, 1, V \psi\right)=-\left(\varphi, \partial_{1} q^{1} V \psi\right)=-(\varphi, V \psi)-\left(\varphi, q^{1} V_{, 1} \psi\right)-\left(\varphi, q^{1} V \psi, 1\right)
$$

we finally obtain that

$$
\mathcal{Q}(\varphi, \psi)=\left(\varphi, j, G^{1 j} \psi_{, 1}\right)+\left(\varphi, 1, G^{1 j} \psi_{, j}\right)-\left(\varphi_{, i}, q^{1} G_{, 1}^{i j} \psi_{, j}\right)-\left(\varphi, q^{1} V_{, 1} \psi\right)
$$

This implies that $\mathcal{Q}$ restricted to $\mathcal{D}(H)_{\mathrm{c}}$ is continuous for the topology induced by $\mathcal{H}_{0}^{1}(\Omega)$. Now, $\mathcal{D}(H)_{\mathrm{c}}$ is dense in $\mathcal{H}_{0}^{1}(\Omega)$ by Lemma 2.6 (ii). Thus, $\mathcal{Q}$ defines (by continuous extension) an operator in $\mathscr{B}\left(\mathcal{H}_{0}^{1}(\Omega), \mathcal{H}^{-1}(\Omega)\right)$, which we shall denote $i[H, A]$. Furthermore, using (2.9), we obtain (2.8) in $\mathscr{B}\left(\mathcal{H}_{0}^{1}(\Omega), \mathcal{H}^{-1}(\Omega)\right)$.

\subsubsection{Strict Mourre estimate for the free Hamiltonian}

Now we prove that $H_{0}$ is of class $C^{\infty}(A)$ and $A$ strictly conjugate to it on $\mathbb{R} \backslash \mathcal{T}$. So, let us first recall the following definition [2] Sec. 7.2.1 \& 7.2.2]:

Definition 2.8. Let $A, H$ be self-adjoint operators in a Hilbert space $\mathcal{H}$ with $H$ of class $C^{1}(A)$. Furthermore, if $S, T \in \mathscr{B}(\mathcal{H})$, we write $S \gtrsim T$ if there exists $K \in \mathcal{K}(\mathcal{H})$ so that $S \geq T+K$. Then, $\forall \lambda \in \mathbb{R}$,

$$
\begin{aligned}
& \varrho_{H}^{A}(\lambda):=\sup \left\{a \in \mathbb{R}: \exists \varepsilon>0 \text { s.t. } E^{H}(\lambda ; \varepsilon) i[H, A] E^{H}(\lambda ; \varepsilon) \geq a E^{H}(\lambda ; \varepsilon)\right\}, \\
& \widetilde{\varrho}_{H}^{A}(\lambda):=\sup \left\{a \in \mathbb{R}: \exists \varepsilon>0 \text { s.t. } E^{H}(\lambda ; \varepsilon) i[H, A] E^{H}(\lambda ; \varepsilon) \gtrsim a E^{H}(\lambda ; \varepsilon)\right\}
\end{aligned}
$$

where $E^{H}(\lambda ; \varepsilon):=E^{H}((\lambda-\varepsilon, \lambda+\varepsilon))$ designates the spectral projection of $H$ for the interval $(\lambda-\varepsilon, \lambda+\varepsilon)$.

We also need the following natural generalisation of [5, Thm. 3.4].

Theorem 2.9. Let $H_{1}, H_{2}$ be two self-adjoint, bounded from below operators in the Hilbert spaces $\mathcal{H}_{1}, \mathcal{H}_{2}$. Assume that $A_{\jmath}, \jmath=1,2$, is a self-adjoint operator in $\mathcal{H}_{\jmath}$ such that $H_{\jmath}$ is of class $C^{k}\left(A_{\jmath}\right), k \in(\mathbb{N} \backslash\{0\}) \cup\{+\infty\}$. Let $H:=H_{1} \otimes 1+1 \otimes H_{2}$ and $A:=A_{1} \otimes 1+1 \otimes A_{2}$, which are self-adjoint operators in $\mathcal{H}_{1} \otimes \mathcal{H}_{2}$. Then $H$ is of class $C^{k}(A)$ and $\forall \lambda \in \mathbb{R}:$

$$
\varrho_{H}^{A}(\lambda)=\inf _{\lambda=\lambda_{1}+\lambda_{2}}\left[\varrho_{H_{1}}^{A_{1}}\left(\lambda_{1}\right)+\varrho_{H_{2}}^{A_{2}}\left(\lambda_{2}\right)\right]
$$

Corollary 2.10. $H_{0} \in C^{\infty}(A)$ and

$$
\forall \lambda \in \mathbb{R}, \quad \varrho_{H_{0}}^{A}(\lambda)=\left\{\begin{array}{lll}
2 \rho(\lambda) & \text { if } & \lambda \geq \nu_{1} \\
+\infty & \text { if } & \lambda<\nu_{1}
\end{array}\right.
$$

where $\rho(\lambda):=\lambda-\sup \{\zeta \in \mathcal{T}: \zeta \leq \lambda\}$ is strictly positive on $\mathbb{R} \backslash \mathcal{T}$. 
Proof. $A_{1}:=A_{\|}, A_{2}:=0$ are self-adjoint in $L^{2}(\mathbb{R})$, respectively $L^{2}(\omega) . H_{1}:=p_{1}^{2}$, $H_{2}:=-\Delta_{\mathrm{D}}^{\omega}$ are self-adjoint, bounded from below in $L^{2}(\mathbb{R})$, respectively $L^{2}(\omega)$. Clearly, [2] Ex. 6.2.8] $p_{1}^{2} \in C^{\infty}\left(A_{\|}\right)$and $-\Delta_{\mathrm{D}}^{\omega} \in C^{\infty}(0)$. The first part of the claim and (2.10) then follows from Theorem 2.9 The expression for $\rho(\lambda)$ is a direct consequence of the respective behaviours of [2] Sec. 7.2.1] $\varrho_{p_{1}^{2}}^{A_{11}}$ and $\varrho_{-\Delta_{\mathrm{D}}^{\omega}}^{0}$ :

$$
\left[\begin{array}{c}
\varrho_{p_{1}^{2}}^{A_{11}}\left(\lambda_{1}\right) \\
\varrho_{-\Delta_{\mathrm{D}}^{\omega}}^{0}\left(\lambda_{2}\right)
\end{array}\right]= \begin{cases}{\left[\begin{array}{c}
2 \lambda_{1} \\
+\infty
\end{array}\right] \text { if }} & {\left[\begin{array}{c}
\lambda_{1} \geq 0 \\
\lambda_{1}<0
\end{array}\right]} \\
{\left[\begin{array}{c}
0 \\
+\infty
\end{array}\right] \quad \text { if }} & {\left[\begin{array}{c}
\lambda_{2} \in \mathcal{T} \\
\lambda_{2} \in \mathbb{R} \backslash \mathcal{T}
\end{array}\right] .}\end{cases}
$$

\subsubsection{Regularity of the Hamiltonian}

In order to prove the regularity of $H$, we need two technical lemmas.

Lemma 2.11. $\forall z \in \mathbb{R} \backslash \sigma(H), \forall \vartheta \leq 1$, one has

(i) $\left[R(z),\left\langle q^{1}\right\rangle^{\vartheta}\right] \in \mathscr{B}\left(\mathcal{H}(\Omega), \mathcal{H}_{0}^{1}(\Omega)\right)$,

(ii) $\forall i \in\{1, \ldots, d\},\left[R(z),\left\langle q^{1}\right\rangle^{\vartheta}\right] \partial_{i} \in \mathscr{B}\left(\mathcal{H}(\Omega), \mathcal{H}_{0}^{1}(\Omega)\right)$.

This is established by adapting the proof of [3] Lemma 4.3] while next Lemma follows from the use of [3, Proof of Prop. 4.2].

Lemma 2.12. Let $S \in \mathscr{B}(\mathcal{H}(\Omega))$ be self-adjoint and $\vartheta \in(0,1]$, then

$$
\left\langle q^{1}\right\rangle^{\vartheta} S \in \mathscr{B}\left(\mathcal{H}(\Omega), \mathcal{H}^{\vartheta}(\mathbb{R}) \otimes L^{2}(\omega)\right) \Longrightarrow S \in C^{\vartheta}(A) .
$$

(Note that the proof involves principally two facts. First, $S \in \mathscr{B}\left(\mathcal{H}(\Omega), \mathcal{D}\left(|A|^{\vartheta}\right)\right.$ ) implies $S \in C^{\vartheta}(A)$. Second, the continuous imbedding $\mathcal{H}_{\vartheta}^{\vartheta}(\mathbb{R}) \subseteq \mathcal{D}\left(\left|A_{\|}\right|^{\vartheta}\right)$, which follows by real interpolation [2] Sec. 2.7] from the continuous imbedding $\mathcal{H}_{1}^{1}(\mathbb{R}) \subseteq \mathcal{D}\left(\left|A_{||}\right|\right)$.)

Remark 2.13. The facts that $i[H, A] \in \mathscr{B}\left(\mathcal{H}_{0}^{1}(\Omega), \mathcal{H}^{-1}(\Omega)\right)$ and that $\mathcal{H}_{0}^{1}(\Omega)$ is stable under $\left\{\mathrm{e}^{i A t}\right\}_{t \in \mathbb{R}}$ imply [2] Sec. 6.3] that $H \in C^{1}(A)$.

Proposition 2.14. $\exists \vartheta \in(0,1]$ such that $H \in C^{1+\vartheta}(A)$.

Proof. We show that each term appearing in the expression for $B:=i[H, A]$ is at least of class $C^{\gamma}(A)$ for a certain $\gamma \in(0,1]$.

Consider first $B_{1}:=-\partial_{j} G^{j 1} \partial_{1}-\partial_{1} G^{1 j} \partial_{j}$. An explicit calculation (analogous to that of the proof of Proposition 2.7) implies that

$$
i\left[B_{1}, A\right]=-2 \partial_{1} G^{11} \partial_{1}-\partial_{1} G^{1 j} \partial_{j}-\partial_{j} G^{j 1} \partial_{1}+\partial_{j} q^{1} G_{, 1}^{j 1} \partial_{1}+\partial_{1} q^{1} G_{, 1}^{1 j} \partial_{j}
$$


as operators in $\mathscr{B}\left(\mathcal{H}_{0}^{1}(\Omega), \mathcal{H}^{-1}(\Omega)\right)$. Thus, $B_{1} \in C^{1}(A)$ by Remark 2.13

Let $z \in \mathbb{R} \backslash \sigma(H)$. As a consequence of the fact that $H \in C^{1}(A)$, one can interpret $i[A, R(z)]$ as the product of [2] Sec. 6.2.2] three bounded operators, viz. $R(z): \mathcal{H}(\Omega) \rightarrow$ $\mathcal{D}(H), B: \mathcal{D}(H) \rightarrow \mathcal{D}(H)^{*}$ and $R(z): \mathcal{D}(H)^{*} \rightarrow \mathcal{H}(\Omega)$. Thus, using Proposition 2.7 one can write as an operator identity in $\mathscr{B}(\mathcal{H}(\Omega))$

$$
\begin{aligned}
i[A, R(z)]=R(z) B R(z)= & R(z) B_{1} R(z)+R(z) \partial_{i} q^{1} G_{, 1}^{i j} \partial_{j} R(z) \\
& -R(z) q^{1} V_{, 1} R(z) .
\end{aligned}
$$

Since the first term has already been shown to be bounded, it is enough to prove that the second and third terms on the r.h.s. are of class $C^{\gamma}(A)$ for some $\gamma \in(0,1]$.

We employ Lemma2.12 with $\vartheta:=\min \left\{\vartheta_{1}, \vartheta_{2}\right\}$ in order to deal with both terms. Using some commutation relations, we get

$$
\begin{aligned}
\left\langle q^{1}\right\rangle^{\vartheta} R(z) \partial_{i} q^{1} G_{, 1}^{i j} \partial_{j} R(z)= & R(z) \partial_{i}\left\langle q^{1}\right\rangle^{\vartheta} q^{1} G^{i j}{ }_{1} \partial_{j} R(z) \\
& -\left[R(z),\left\langle q^{1}\right\rangle^{\vartheta}\right] \partial_{i} q^{1} G^{i j}{ }_{1} \partial_{j} R(z) \\
& -R(z)\left[\partial_{i},\left\langle q^{1}\right\rangle^{\vartheta}\right] q^{1} G^{i j}{ }_{, 1} \partial_{j} R(z) .
\end{aligned}
$$

Under Assumption 2.13 , the first term on the r.h.s. is in $\mathscr{B}\left(\mathcal{H}(\Omega), \mathcal{H}_{0}^{1}(\Omega)\right)$. The second and the last one are in $\mathscr{B}\left(\mathcal{H}(\Omega), \mathcal{H}_{0}^{1}(\Omega)\right)$ by Lemma 2.11 (ii) and the boundedness of $\left\langle q^{1}\right\rangle_{, 1}^{\vartheta}$, respectively. Moreover,

$$
\left\langle q^{1}\right\rangle^{\vartheta} R(z) q^{1} V_{, 1} R(z)=R(z)\left\langle q^{1}\right\rangle^{\vartheta} q^{1} V_{, 1} R(z)+\left[\left\langle q^{1}\right\rangle^{\vartheta}, R(z)\right] q^{1} V_{, 1} R(z)
$$

is in $\mathscr{B}\left(\mathcal{H}(\Omega), \mathcal{H}_{0}^{1}(\Omega)\right)$ by Assumption 2.23 and Lemma 2.11(i). Thus, all the terms in the expression of $B$ are at least of class $C^{\vartheta}(A)$. This implies the claim.

\subsubsection{The main result}

Proposition 2.15. $\forall \lambda \in \mathbb{R} \backslash \mathcal{T}, \widetilde{\varrho}_{H}^{A}(\lambda)>0$.

Proof. Corollary 2.10 and Proposition 2.14 imply that both $H_{0}$ and $H$ are of class $C_{u}^{1}(A)$. Furthermore, $R(i)-R_{0}(i)$ is compact by Proposition 2.2 with the result that $\widetilde{\varrho}_{H}^{A}=$ $\widetilde{\varrho}_{H_{0}}^{A}$ due to [2] Thm. 7.2.9]. Finally, since [2] Prop. 7.2.6] $\widetilde{\varrho}_{H_{0}}^{A} \geq \varrho_{H_{0}}^{A}$, we can conclude using Corollary 2.10

Summing up, we result in the following spectral properties of $H$.

Theorem 2.16. Let $\omega$ be a bounded open connected set in $\mathbb{R}^{d-1}, d \geq 2$, and denote by $\mathcal{T}$ the set of eigenvalues of $-\Delta_{\mathrm{D}}^{\omega}$. Let $H$ be the operator 11.3 with $\Omega:=\mathbb{R} \times \omega$, subject to Dirichlet boundary conditions, and satisfying Assumptions 2.1 and 2.2 Then

(i) $\sigma_{\text {ess }}(H)=[\kappa, \infty)$, where $\kappa:=\inf \mathcal{T}$,

(ii) $\sigma_{\mathrm{sc}}(H)=\varnothing$, 
(iii) $\sigma_{\mathrm{p}}(H) \cup \mathcal{T}$ is closed and countable,

(iv) $\sigma_{\mathrm{p}}(H) \backslash \mathcal{T}$ is composed of finitely degenerated eigenvalues, which can accumulate at the points of $\mathcal{T}$ only,

Proof. The claim (i) is included in Proposition 2.2 Since $A$ is conjugate to $H$ on $\mathbb{R} \backslash \mathcal{T}$ by Proposition 2.15, the assertions (ii)-(iv) follow by the abstract conjugate operator method [2] Thm. 7.4.2].

To conclude this section, let us remark that Assumptions 2.13 and 2.23 could be weakened. Firstly, we recall that the situation with $V=0$ and $G=\rho 1, \rho$ being a realvalued function greater than a strictly positive constant, is investigated in [3] [10] where the authors admit local singularities of $\rho$. More specifically, one assumes that $\rho=\rho_{\mathrm{s}}+\rho_{\ell}$, where $\rho_{\ell}$ is the part satisfying a condition analogous to Assumption 2.1] 3, while $\rho_{\mathrm{s}}$ need not be differentiable. (In [3], $\operatorname{supp}\left(\rho_{\mathrm{s}}\right)$ is assumed to be compact. The result of [10] is better in the sense that $\rho_{s}$ is only supposed to be a short-range perturbation there. However, this requires strengthening of the condition analogous to Assumption 2.12 about the decay of $\rho$ at infinity.) Secondly, the optimal conditions one has to impose on the potential of a Schrödinger operator are known [6, 2].

\section{Curved tubes}

In this part, we use Theorem 2.16 in order to find geometric sufficient conditions which guarantee that the spectral results of the theorem hold true for curved tubes.

\subsection{Geometric preliminaries}

\subsubsection{The reference curve}

Given $d \geq 2$, let $p: \mathbb{R} \rightarrow \mathbb{R}^{d}$ be a regular unit-speed smooth (i.e., $C^{\infty}$-smooth) curve satisfying the following hypothesis.

Assumption 3.1. There exists a collection of $d$ smooth mappings $e_{i}: \mathbb{R} \rightarrow \mathbb{R}^{d}$ with the following properties:

1. $\forall i, j \in\{1, \ldots, d\}, \forall s \in \mathbb{R}, e_{i}(s) \cdot e_{j}(s)=\delta_{i j}$,

2. $\forall i \in\{1, \ldots, d-1\}, \forall s \in \mathbb{R}$, the $i^{\text {th }}$ derivative $p^{(i)}(s)$ of $p(s)$ lies in the span of $e_{1}(s), \ldots, e_{i}(s)$,

3. $e_{1}=\dot{p}$,

4. $\forall s \in \mathbb{R},\left\{e_{1}(s), \ldots, e_{d}(s)\right\}$ has the positive orientation,

5. $\forall i \in\{1, \ldots, d-1\}, \forall s \in \mathbb{R}, \dot{e}_{i}(s)$ lies in the span of $e_{1}(s), \ldots, e_{i+1}(s)$.

Here and in the sequel, "." denotes the inner product in $\mathbb{R}^{d}$. 
Remark 3.1. A vector field with the property 1 is called a moving frame along $p$ and it is a Frenet frame if it satisfies 2 in addition, cf. [22] Sec. 1.2]. A sufficient condition to ensure the existence of the frame of Assumption 3.1 is to require that [22] Prop. 1.2.2], for all $s \in \mathbb{R}$, the vectors $\dot{p}(s), p^{(2)}(s), \ldots, p^{(d-1)}(s)$ are linearly independent. This is always satisfied if $d=2$. However, we do not assume a priori the above non-degeneracy condition for $d \geq 3$ because it excludes the curves such that, for some open $I \subseteq \mathbb{R}, p\lceil I$ lies in a lower-dimensional subspace of $\mathbb{R}^{d}$.

The properties of $\left\{e_{1}, \ldots, e_{d}\right\}$ summarised in Assumption 3.1 yield [22] Sec. 1.3] the Serret-Frenet formulae,

$$
\dot{e}_{i}=\mathcal{K}_{i}^{j} e_{j}
$$

with $\mathcal{K} \equiv\left(\mathcal{K}_{i}^{j}\right)$ being a skew-symmetric $d \times d$ matrix defined by

$$
\mathcal{K}:=\left(\begin{array}{cccc}
0 & \kappa_{1} & & 0 \\
-\kappa_{1} & \ddots & \ddots & \\
& \ddots & \ddots & \kappa_{d-1} \\
0 & & -\kappa_{d-1} & 0
\end{array}\right) .
$$

Here $\kappa_{i}$ is called the $i^{\text {th }}$ curvature of $p$. Under our Assumption 3.1 the curvatures are smooth functions of the arc-length parameter $s \in \mathbb{R}$.

\subsubsection{The appropriate moving frame}

In this subsection, we introduce another moving frame along $p$, which better reflects the geometry of the curve, and will be used later to define a tube about it. We shall refer to it as the Tang frame because it is a natural generalisation of the Tang frame known from the theory of 3-dimensional waveguides [31, 17] 11]. Our construction follows the generalisation introduced in [8]. equations

Let the $(d-1) \times(d-1)$ matrix $\left(\mathcal{R}_{\mu}^{\nu}\right)$ be defined by the system of differential

$$
\dot{\mathcal{R}}_{\mu}^{\nu}+\mathcal{R}_{\mu}^{\alpha} \mathcal{K}_{\alpha}^{\nu}=0
$$

with $\left(\mathcal{R}_{\mu}^{\nu}\left(s_{0}\right)\right)$ being a rotation matrix in $\mathbb{R}^{d-1}$ for some $s_{0} \in \mathbb{R}$ as initial condition, i.e.,

$$
\operatorname{det}\left(\mathcal{R}_{\mu}^{\nu}\left(s_{0}\right)\right)=1 \quad \text { and } \quad \delta_{\alpha \beta} \mathcal{R}_{\mu}^{\alpha}\left(s_{0}\right) \mathcal{R}_{\nu}^{\beta}\left(s_{0}\right)=\delta_{\mu \nu} .
$$

The solution of 3.3 exists and is smooth by standard arguments in the theory of differential equations (cf. [25] Sec. 4]). Furthermore, the conditions (3.4) are satisfied for all $s_{0} \in \mathbb{R}$. Indeed, by means of Liouville's formula [25. Thm. 4.7.1] and $\operatorname{tr}(\mathcal{K})=0$, one checks that $\operatorname{det}\left(\mathcal{R}_{\mu}^{\nu}\right)=1$ identically, while the validity of the second condition for all $s_{0} \in \mathbb{R}$ is obtained via the skew-symmetry of $\mathcal{K}$ :

$$
\left(\delta_{\alpha \beta} \mathcal{R}_{\mu}^{\alpha} \mathcal{R}_{\nu}^{\beta}\right)^{\cdot}=-\mathcal{R}_{\mu}^{\alpha}\left(\delta_{\gamma \beta} \mathcal{K}_{\alpha}^{\gamma}+\delta_{\alpha \gamma} \mathcal{K}_{\beta}^{\gamma}\right) \mathcal{R}_{\nu}^{\beta}=0 .
$$


We set

$$
\mathcal{R} \equiv\left(\mathcal{R}_{i}^{j}\right):=\left(\begin{array}{cc}
1 & 0 \\
0 & \left(\mathcal{R}_{\mu}^{\nu}\right)
\end{array}\right)
$$

and introduce the Tang frame as the moving frame $\left\{\tilde{e}_{1}, \ldots, \tilde{e}_{d}\right\}$ along $p$ defined by

$$
\tilde{e}_{i}:=\mathcal{R}_{i}^{j} e_{j}
$$

Combining 3.1 with 3.3, one easily finds

$$
\dot{\tilde{e}}_{1}=\kappa_{1} e_{2} \quad \text { and } \quad \dot{\tilde{e}}_{\mu}=\mathcal{R}_{\mu}^{\alpha} \mathcal{K}_{\alpha}^{1} e_{1}=-\kappa_{1} \mathcal{R}_{\mu}^{2} e_{1} .
$$

The interest of the Tang frame will appear in the following subsection.

\subsubsection{The tube}

Let $\omega$ be a bounded open connected set in $\mathbb{R}^{d-1}$. Without loss of generality, we assume that $\omega$ is translated so that its centre of mass is at the origin. Let $\Omega:=\mathbb{R} \times \omega$ be a straight tube. We define the curved tube $\Gamma$ of the same cross-section $\omega$ about $p$ as the image of the mapping

$$
\mathscr{L}: \Omega \rightarrow \mathbb{R}^{d}, \quad\left(s, u^{2}, \ldots, u^{d}\right) \mapsto p(s)+\tilde{e}_{\mu}(s) u^{\mu},
$$

i.e., $\Gamma:=\mathscr{L}(\Omega)$.

As already mentioned in Introduction, the shape of the curved tube $\Gamma$ of crosssection $\omega$ about $p$ depends on the choice of rotations $\left(\mathcal{R}_{\mu}^{\nu}\right)$ in $(3.5$, unless $\omega$ is rotation invariant. As usual in the theory of quantum waveguides (see, e.g., [11] 8]), we restrict ourselves to the technically most advantageous choice determined by (3.3), i.e., when the cross-section $\omega$ rotates along $p$ w.r.t. the Tang frame (another choice can be found in [14]).

We write $u \equiv\left(u^{2}, \ldots, u^{d}\right)$, define $a:=\sup _{u \in \omega}|u|$ and always assume

\section{Assumption 3.2.}

1. $\kappa_{1} \in L^{\infty}(\mathbb{R})$ and $a\left\|\kappa_{1}\right\|_{\infty}<1$,

\section{2. $\Gamma$ does not overlap itself.}

Then, the mapping $\mathscr{L}: \Omega \rightarrow \Gamma$ is a diffeomorphism. Indeed, by virtue of the inverse function theorem, the first condition guarantees that it is a local diffeomorphism which is global through the injectivity induced by the second condition. Consequently, $\mathscr{L}^{-1}$ determines a system of global (geodesic or Fermi) "coordinates" $(s, u)$. At the same time, the tube $\Gamma$ can be identified with the Riemannian manifold $(\Omega, g)$, where $g \equiv\left(g_{i j}\right)$ is the metric tensor induced by the immersion (3.7), that is $g_{i j}:=\mathscr{L}_{, i} \cdot \mathscr{L}_{, j}$. The formulae 3.6) yield

$$
g=\operatorname{diag}\left(h^{2}, 1, \ldots, 1\right) \quad \text { with } \quad h(s, u):=1+u^{\mu} \mathcal{R}_{\mu}^{\alpha}(s) \mathcal{K}_{\alpha}^{1}(s) .
$$

Note that the metric tensor 3.8 is diagonal due to our special choice of the "transverse" frame $\left\{\tilde{e}_{2}, \ldots, \tilde{e}_{d}\right\}$, which is the advantage of the Tang frame.

We set $|g|:=\operatorname{det}(g)=h^{2}$, which defines through $\mathrm{d} v:=h(s, u) \mathrm{d} s \mathrm{~d} u$ the volume element of $\Gamma$; here $\mathrm{d} u$ denotes the $(d-1)$-dimensional Lebesgue measure in $\omega$. 
Remark 3.2 (Low-dimensional examples). When $d=2$, the cross-section $\omega$ is just the interval $(-a, a)$, the curve $p$ has only one curvature $\kappa:=\kappa_{1}$, the rotation matrix $\left(\mathcal{R}_{\mu}^{\nu}\right)$ equals (the scalar) 1 and

$$
h(s, u)=1-\kappa(s) u .
$$

If $d=3$, it is convenient to make the Ansatz

$$
\left(\mathcal{R}_{\mu}^{\nu}\right)=\left(\begin{array}{cc}
\cos \alpha & -\sin \alpha \\
\sin \alpha & \cos \alpha
\end{array}\right)
$$

where $\alpha$ is a real-valued differentiable function. Then, it is easy to see that (3.3) reduces to the differential equation $\dot{\alpha}=\tau$, where $\tau$ is the torsion of $p$, i.e., one puts $\kappa:=\kappa_{1}$ and $\tau:=\kappa_{2}$. Choosing $\alpha$ as an integral of $\tau$, we can write

$$
h(s, u)=1-\kappa(s)\left[u^{2} \cos \alpha(s)+u^{3} \sin \alpha(s)\right] .
$$

Remark 3.3 (On Assumption 3.2). If $p$ were a compact embedded curve, then Assumption 3.2 could always be achieved for sufficiently small a. In general, however, one cannot exclude self-intersections of the tube using the local geometry of an embedded curve $p$ only. One way to avoid this disadvantage would be to consider $(\Omega, g)$ as an abstract Riemannian manifold where only the curve $p$ is embedded in $\mathbb{R}^{d}$. Nonetheless, in the present paper, we prefer to assume Assumption 3.22 a priori because $\Gamma$ does not have a physical meaning if it is self-intersecting. Finding global geometric conditions on p ensuring the validity of Assumption 3.2 2 is an interesting question, which is beyond the scope of the present paper, however.

\subsection{The Laplacian}

Our object of interest is the Dirichlet Laplacian (1.2), with $\Gamma$ defined by 3.7). We construct it as follows. Using the diffeomorphism (3.7), we identify the Hilbert space $L^{2}(\Gamma)$ with $L^{2}(\Omega, \mathrm{d} v)$ and consider on the latter the Dirichlet form

$$
\widetilde{Q}(\varphi, \psi):=\int_{\Omega} \overline{\varphi_{, i}} g^{i j} \psi_{, j} \mathrm{~d} v, \quad \varphi, \psi \in \mathcal{D}(\widetilde{Q}):=\mathcal{H}_{0}^{1}(\Omega, \mathrm{d} v),
$$

where $\left(g^{i j}\right):=g^{-1}$. The form $\widetilde{Q}$ is clearly densely defined, non-negative, symmetric and closed on its domain. Consequently, there exists a unique non-negative self-adjoint operator $\widetilde{H}$ satisfying $\mathcal{D}(\widetilde{H}) \subset \mathcal{D}(\widetilde{Q})$ associated with $\widetilde{Q}$. We have

$$
\begin{aligned}
\widetilde{H} \psi & =-|g|^{-1 / 2} \partial_{i}|g|^{1 / 2} g^{i j} \partial_{j} \psi, \\
\psi \in \mathcal{D}(\widetilde{H}) & =\left\{\psi \in \mathcal{H}_{0}^{1}(\Omega, \mathrm{d} v): \partial_{i}|g|^{1 / 2} g^{i j} \partial_{j} \psi \in L^{2}(\Omega, \mathrm{d} v)\right\} .
\end{aligned}
$$

That is, $\widetilde{H}$ is the Laplacian (1.2) expressed in the coordinates $(s, u)$.

In order to apply Theorem 2.16 we transform $\widetilde{H}$ into a unitarily equivalent operator $H$ of the form (1.3) acting on the Hilbert space $\mathcal{H}(\Omega):=L^{2}(\Omega)$, without the additional 
weight $|g|^{1 / 2}$ in the volume element. This is achieved by means of the unitary mapping $\mathcal{U}: L^{2}(\Omega, \mathrm{d} v) \rightarrow \mathcal{H}(\Omega), \psi \mapsto|g|^{1 / 4} \psi$. Defining $H:=\mathcal{U} \widetilde{H} \mathcal{U}^{-1}$, one has

$$
\begin{aligned}
H \psi & =-|g|^{-1 / 4} \partial_{i}|g|^{1 / 2} g^{i j} \partial_{j}|g|^{-1 / 4} \psi, \\
\psi \in \mathcal{D}(H) & =\left\{\psi \in \mathcal{H}_{0}^{1}(\Omega): \partial_{i}|g|^{1 / 2} g^{i j} \partial_{j}|g|^{-1 / 4} \psi \in L^{2}(\Omega)\right\} .
\end{aligned}
$$

Commuting $|g|^{-1 / 4}$ with the gradient components in the expression for $H$, we obtain on $\mathcal{D}(H)$

$$
H=-\partial_{i} g^{i j} \partial_{j}+V
$$

where

$$
V:=-\frac{5}{4} \frac{\left(h_{, 1}\right)^{2}}{h^{4}}+\frac{1}{2} \frac{h_{, 11}}{h^{3}}-\frac{1}{4} \frac{\delta^{\mu \nu} h_{, \mu} h_{, \nu}}{h^{2}}+\frac{1}{2} \frac{\delta^{\mu \nu} h_{, \mu \nu}}{h} .
$$

Actually, 3.14 with 3.15 is a general formula valid for any smooth metric of the form $g=\operatorname{diag}\left(h^{2}, 1, \ldots, 1\right)$. In our special case with $h$ given by 3.8 , we find that $h_{, \mu \nu}=0, \delta^{\mu \nu} h_{, \mu} h_{, \nu}=\delta^{\alpha \beta} \mathcal{K}_{\alpha}^{1} \mathcal{K}_{\beta}^{1}$ by (3.4), while (3.3) gives

$$
\begin{aligned}
h_{, 1}(\cdot, u) & =u^{\mu} \mathcal{R}_{\mu}^{\alpha}\left(\dot{\mathcal{K}}_{\alpha}^{1}-\mathcal{K}_{\alpha}^{\beta} \mathcal{K}_{\beta}^{1}\right) \\
h_{, 11}(\cdot, u) & =u^{\mu} \mathcal{R}_{\mu}^{\alpha}\left(\ddot{\mathcal{K}}_{\alpha}^{1}-\dot{\mathcal{K}}_{\alpha}^{\beta} \mathcal{K}_{\beta}^{1}-2 \mathcal{K}_{\alpha}^{\beta} \dot{\mathcal{K}}_{\beta}^{1}+\mathcal{K}_{\alpha}^{\beta} \mathcal{K}_{\beta}^{\gamma} \mathcal{K}_{\gamma}^{1}\right) .
\end{aligned}
$$

\subsection{Results}

It remains to impose decay conditions on the curvatures of $p$ (and their derivatives) in order that the operator (3.14) satisfies Assumption 2.1 and Assumption 2.2.

Let us first consider the more general situation where the matrix $\left(g^{i j}\right)$ is equal to $\operatorname{diag}\left(h^{-2}, 1, \ldots, 1\right)$ with the explicit dependence of $h$ on $s$ and $u$ not specified. One shows that it is sufficient to impose the following hypotheses.

Assumption 3.3. Uniformly for $u \in \omega$,

1. $h(s, u) \longrightarrow 1$ as $|s| \rightarrow \infty$,

2. $h_{, 11}(s, u),\left(\delta^{\mu \nu} h_{, \mu} h_{, \nu}\right)(s, u), \delta^{\mu \nu} h_{, \mu \nu}(s, u) \longrightarrow 0$ as $|s| \rightarrow \infty$,

3. $\exists \vartheta \in(0,1]$ s.t.

$$
h_{, 1}(s, u), h_{, 111}(s, u),\left(\delta^{\mu \nu} h_{, \mu} h_{, \nu}\right)_{, 1}(s, u), \delta^{\mu \nu} h_{, 1 \mu \nu}(s, u)=\mathcal{O}\left(|s|^{-(1+\vartheta)}\right) .
$$

Indeed, the first hypothesis supplies Assumption 2.12, while Assumption 2.11 is fulfilled due to basic Assumption 3.2 Next, since $h$ is a smooth function, Assumption 3.32 together with the behaviour of $h_{, 1}$ in Assumption 3.33 are sufficient to ensure both Assumption 2.2 1 and Assumption 2.2 2. It is also clear that the asymptotic behaviour of $h_{, 1}$ 
in Assumption 3.33 supplies Assumption 2.13. Assumption 2.14 holds true due to Assumption 2.13 and the particular form of $\left(g^{i j}\right)$. It remains to check Assumption 2.2 3 . This is easily done by calculating the derivative of the potential 3.15 :

$$
\begin{aligned}
V_{, 1}=5 & \frac{\left(h_{, 1}\right)^{3}}{h^{5}}-4 \frac{h_{, 1} h_{, 11}}{h^{4}}+\frac{h_{, 111}}{2 h^{3}} \\
& +\frac{\delta^{\mu \nu}}{2}\left(\frac{h_{, 1} h_{, \mu} h_{, \nu}}{h^{3}}-\frac{h_{, 1} h_{, \mu \nu}+h_{, 1 \mu} h_{, \nu}}{h^{2}}+\frac{h_{, 1 \mu \nu}}{h}\right) .
\end{aligned}
$$

With $h$ given by 3.8 , we find in addition to 3.16 that $h_{, 1 \mu \nu}=0$ and

$$
\begin{aligned}
\left(\delta^{\mu \nu} h_{, \mu} h_{, \nu}\right)_{, 1}= & 2 \delta^{\alpha \beta} \dot{\mathcal{K}}_{\alpha}^{1} \mathcal{K}_{\beta}^{1} \\
h_{, 111}(\cdot, u)= & u^{\mu} \mathcal{R}_{\mu}^{\alpha}\left(\dddot{\mathcal{K}}_{\alpha}^{1}-\ddot{\mathcal{K}}_{\alpha}^{\beta} \mathcal{K}_{\beta}^{1}-3 \mathcal{K}_{\alpha}^{\beta} \ddot{\mathcal{K}}_{\beta}^{1}-3 \dot{\mathcal{K}}_{\alpha}^{\beta} \dot{\mathcal{K}}_{\beta}^{1}+\dot{\mathcal{K}}_{\alpha}^{\beta} \mathcal{K}_{\beta}^{\gamma} \mathcal{K}_{\gamma}^{1}\right. \\
& \left.+2 \mathcal{K}_{\alpha}^{\beta} \dot{\mathcal{K}}_{\beta}^{\gamma} \mathcal{K}_{\gamma}^{1}+3 \mathcal{K}_{\alpha}^{\beta} \mathcal{K}_{\beta}^{\gamma} \dot{\mathcal{K}}_{\gamma}^{1}-\mathcal{K}_{\alpha}^{\beta} \mathcal{K}_{\beta}^{\gamma} \mathcal{K}_{\gamma}^{\delta} \mathcal{K}_{\delta}^{1}\right) .
\end{aligned}
$$

Since $\left|u^{\mu} \mathcal{R}_{\mu}{ }^{\alpha}\right|<a$, Assumption 3.3 holds true provided we impose the following conditions on the curvatures

\section{Assumption 3.4.}

1. $\forall \alpha \in\{2, \ldots, d\}, \mathcal{K}_{\alpha}^{1}(s), \ddot{\mathcal{K}}_{\alpha}^{1}(s) \longrightarrow 0$ as $|s| \rightarrow \infty$,

2. $\forall \alpha, \beta \in\{2, \ldots, d\}, \mathcal{K}_{\alpha}^{\beta}, \dot{\mathcal{K}}_{\alpha}^{2} \in L^{\infty}(\mathbb{R})$,

3. $\exists \vartheta \in(0,1]$ s.t. $\forall \alpha \in\{2, \ldots, d\}$,

$$
\dot{\mathcal{K}}_{\alpha}^{1}(s), \dddot{\mathcal{K}}_{\alpha}^{1}(s), \mathcal{K}_{\alpha}^{2}(s), \ddot{\mathcal{K}}_{\alpha}^{2}(s),\left(\dot{\mathcal{K}}_{\alpha}^{\beta} \mathcal{K}_{\beta}^{2}\right)(s),\left(\mathcal{K}_{\alpha}^{\beta} \dot{\mathcal{K}}_{\beta}^{2}\right)(s)=\mathcal{O}\left(|s|^{-(1+\vartheta)}\right) .
$$

Remark 3.4. These conditions reduce to those of Theorem 1.1 provided $d=2$. When $d=3$, it is sufficient to assume the conditions of Theorem 1.1 for the first curvature, and $\dot{\kappa}_{2} \in L^{\infty}(\mathbb{R})$ and $\kappa_{2}(s), \ddot{\kappa}_{2}(s)=\mathcal{O}\left(|s|^{-(1+\vartheta)}\right)$ for some $\vartheta \in(0,1]$.

We conclude this section by applying Theorem 2.16

Theorem 3.5. Let $\Gamma$ be a tube defined via (3.7) about a smooth infinite curve embedded in $\mathbb{R}^{d}$. Suppose Assumptions 3.13 .2 and 3.4 Then all the spectral results (i)-(iv) of Theorem 2.16 hold true for the Dirichlet Laplacian on $L^{2}(\Gamma)$.

\section{Curved strips on surfaces}

In this final section, we investigate the situation where the ambient space is a general Riemannian manifold instead of the Euclidean space $\mathbb{R}^{d}$. We restrict ourselves to $d=2$, i.e., $\Gamma$ is a strip around an infinite curve in an (abstract) two-dimensional surface. We refer to [23] for basic spectral properties of $-\Delta_{D}^{\Gamma}$ and geometric details. 


\subsection{Preliminaries}

Let $\mathcal{A}$ be a smooth connected complete non-compact two-dimensional Riemannian manifold of bounded Gauss curvature $K$. Let $p: \mathbb{R} \rightarrow \mathcal{A}$ be a smooth unit-speed curve embedded in $\mathcal{A}$ with (geodesic) curvature $\kappa$ and denote by $n: \mathbb{R} \rightarrow T_{p(\cdot)} \mathcal{A}$ a smooth unit normal vector field along $p$. Given $a>0$, we consider the straight strip $\Omega:=\mathbb{R} \times(-a, a)$ and define a curved strip $\Gamma$ of same width over $p$ as a $a$-tubular neighbourhood of $p$ in $\mathcal{A}$ by

$$
\Gamma:=\mathscr{L}(\Omega), \quad \text { where } \quad \mathscr{L}:(s, u) \mapsto \exp _{p(s)}(\text { un }(s)) .
$$

Note that $s \mapsto \mathscr{L}(s, u)$ traces the curves parallel to $p$ at a fixed distance $|u|$, while the curve $u \mapsto \mathscr{L}(s, u)$ is a unit-speed geodesic orthogonal to $p$ for any fixed $s$. We always assume

Assumption 4.1. $\mathscr{L}: \Omega \rightarrow \Gamma$ is a diffeomorphism,

Then $\mathscr{L}^{-1}$ determines a system of Fermi "coordinates" $(s, u)$, i.e., the geodesic coordinates based on $p$. The metric tensor of $\Gamma$ in these coordinates acquires [18, Sec. 2.4] the diagonal form

$$
g(s, u)=\operatorname{diag}\left(h^{2}(s, u), 1\right),
$$

where $h$ is a smooth function satisfying the Jacobi equation

$$
h_{, 22}+K h=0 \quad \text { with } \quad\left\{\begin{array}{l}
h(\cdot, 0)=1 \\
h_{, 2}(\cdot, 0)=-\kappa .
\end{array}\right.
$$

Here $K$ and $\kappa$ are considered as functions of the Fermi coordinates (the sign of $\kappa$ being uniquely determined up to the re-parameterisation $s \mapsto-s$ or the choice of $n$ ). The determinant of the metric tensor, $|g|:=\operatorname{det}(g)=h^{2}$, defines through $\mathrm{d} v:=h(s, u) \mathrm{d} s \mathrm{~d} u$ the area element of the strip.

Assuming that the metric $g$ is uniformly elliptic in the sense that

Assumption 4.2. $\exists c_{ \pm} \in(0, \infty)$ s.t. $\forall(s, u) \in \Omega, c_{-} \leq h(s, u) \leq c_{+}$

holds true, the Dirichlet Laplacian corresponding to $\Gamma$ can be defined in the same way as in Section 3.2 i.e., as the operator $\widetilde{H}$ associated with the form (3.9), satisfying (3.10). At the same time, we may introduce the unitarily equivalent operator $H$ on $L^{2}(\Omega)$ given by (3.12) and satisfying (3.14) with 3.15).

Remark 4.1. If Assumption 4.2 holds true, then the inverse function theorem together with (4.3) yield that Assumption 4.1 is satisfied for all sufficiently small a provided the strip $\Gamma$ does not overlap itself. Assumption 4.2 is satisfied, for instance, if $\Gamma$ is a sufficiently thin strip on a ruled surface, cf. [23, Sec. 7]. 


\subsection{Results}

In view of the more general approach in the beginning of Section 3.3. we see that Assumption 3.3 (with $d=2$ ) guarantees Assumptions 2.1 and 2.2 also in the present case. Applying Theorem 2.16 we obtain, with $\mathcal{T}=\left\{n^{2} \nu_{1}\right\}_{n=1}^{\infty}$ where $\nu_{1}:=\pi^{2} /(2 a)^{2}$, the following result

Theorem 4.2. Let $\Gamma$ be a tubular neighbourhood of radius $a>0$ about a smooth infinite curve, which is embedded in a smooth connected complete non-compact surface of bounded curvature. Suppose Assumptions 4.1 4.2 and 3.3 Then all the spectral results (i)-(iv) of Theorem 2.16 hold true for the Dirichlet Laplacian on $L^{2}(\Gamma)$.

Assume now that the strip is flat in the sense of [23], i.e., the curvature $K$ is equal to zero everywhere on $\Gamma$. Then the Jacobi equation (4.3) has the explicit solution ( $c f .3 .8$ for $d=2$ )

$$
h(s, u)=1-\kappa(s) u
$$

and Assumption 3.3 can be replaced by some conditions on the decay of the curvature $\kappa$ at infinity, namely, we adopt Assumption 3.4 with $\kappa_{1} \equiv \kappa$ and $\mathcal{K}_{\mu}^{\nu}=0$ (cf. the assumptions of Theorem 1.1. At the same time, it easy to see that Assumption 4.1 and 4.2 are satisfied if Assumption 3.2 holds true.

Theorem 4.3 (Flat strips). Let $\Gamma$ be a tubular neighbourhood of radius $a>0$ about a smooth infinite curve of curvature $\kappa$, which is embedded in a smooth connected complete non-compact surface of bounded curvature $K$ such that $K \uparrow \Gamma=0$. Suppose Assumption 3.2 and

1. $\kappa(s), \ddot{\kappa}(s) \longrightarrow 0$ as $|s| \rightarrow \infty$,

2. $\exists \vartheta \in(0,1]$ s.t. $\dot{\kappa}(s), \dddot{\kappa}(s)=\mathcal{O}\left(|s|^{-(1+\vartheta)}\right)$.

Then, all the spectral results (i)-(iv) of Theorem 2.16 hold true for the Dirichlet Laplacian on $L^{2}(\Gamma)$.

\section{Acknowledgements}

One of the authors (D.K.) would like to thank M. Măntoiu and R. Purice for proposing to him to use the conjugate operator method in the theory of quantum waveguides and for many discussions on the topic. We are very grateful to W. Amrein for his careful reading of the present paper and S. Richard for his useful suggestions and comments. This work was partially supported by FCT/POCTI/FEDER, Portugal, GA AS CR grant IAA 1048101 and the Fonds National Suisse.

\section{References}

[1] R. A. Adams, Sobolev spaces, Academic Press, Inc., New York, 1975. 
[2] W. O. Amrein, A. Boutet de Monvel and V. Georgescu, $C_{0}$-groups, commutator methods and spectral theory of $\mathrm{N}$-body Hamiltonians, Progress in Math. Ser., vol. 135, Birkhaüser, 1996.

[3] M. Benbernou, Spectral analysis of the acoustic propagator in a multistratified domain, J. Math. Anal. Appl. 225 (1998), no. 2, 440-460.

[4] D. Borisov, P. Exner, R. Gadyl'shin, and D. Krejčiřík, Bound states in weakly deformed strips and layers, Ann. H. Poincaré 2 (2001), 553-572.

[5] A. Boutet de Monvel-Berthier and V. Georgescu, Graded $C^{*}$-Algebras and ManyBody Perturbation Theory: II The Mourre Estimate, Astérisque 210 (1992), 75-96.

[6] A. Boutet de Monvel-Berthier, V. Georgescu and M. Măntoiu, Locally smooth operators and the limiting absorption principle for $\mathrm{N}$-body Hamiltonians, Rev. Math. Phys. 5 (1993), no. 1, 105-189.

[7] W. Bulla, F. Gesztesy, W. Renger, and B. Simon, Weakly coupled bound states in quantum waveguides, Proc. Amer. Math. Soc. 125 (1997), 1487-1495.

[8] B. Chenaud, P. Duclos, P. Freitas and D. Krejčiřík, Geometrically induced discrete spectrum in curved tubes, Differential Geometry and its Applications, to appear.

[9] H. L. Cycon, R. G. Froese, W. Kirsch and B. Simon, Schrödinger operators with applications to quantum mechanics and global geometry, Springer-Verlag, Berlin Heidelberg, 1987.

[10] Y. Dermenjian, M. Durand and V. Iftimie, Spectral analysis of an acoustic multistratified perturbed cylinder, Commun. in Partial Differential Equations 23 (1998), no. $1 \& 2,141-169$.

[11] P. Duclos and P. Exner, Curvature-induced bound states in quantum waveguides in two and three dimensions, Rev. Math. Phys. 7 (1995), 73-102.

[12] P. Duclos, P. Exner and B. Meller, Exponential bounds on curvature-induced resonances in two-dimensional Dirichlet tube, Helv. Phys. Acta 71 (1998), 133-162.

[13] P. Duclos, P. Exner and P. Štovíček, Curvature-induced resonances in a twodimensional Dirichlet tube, Ann. Inst. H. Poincaré 62 (1995), 81-101.

[14] P. Exner, P. Freitas, and D. Krejčiřík, A lower bound to the spectral threshold in curved tubes, R. Soc. Lond. Proc. Ser. A Math. Phys. Eng. Sci. 460 (2004), no. 2052, 3457-3467.

[15] P. Exner and P. Šeba, Bound states in curved quantum waveguides, J. Math. Phys. 30 (1989), 2574-2580.

[16] P. Exner and S. A. Vugalter, Bound states in a locally deformed waveguide: The critical case, Lett. Math. Phys. 39 (1997), 59-68. 
[17] J. Goldstone and R. L. Jaffe, Bound states in twisting tubes, Phys. Rev. B 45 (1992), 14100-14107.

[18] A. Gray, Tubes, Addison-Wesley Publishing Company, New York, 1990.

[19] N. E. Hurt, Mathematical physics of quantum wires and devices, Kluwer, Dordrecht, 2000.

[20] R. V. Kadison and J. R. Ringrose, Fundamentals of the theory of operator algebras II, Academic Press, Inc., London, 1986.

[21] T. Kato, Perturbation theory for linear operators, Springer-Verlag, Berlin, 1966.

[22] W. Klingenberg, A course in differential geometry, Springer-Verlag, New York, 1978.

[23] D. Krejčiřík, Quantum strips on surfaces, J. Geom. Phys. 45 (2003), no. 1-2, $203-$ 217.

[24] D. Krejčiřík and J. Kříž, On the spectrum of curved quantum waveguides, Publ. RIMS, Kyoto University, to appear.

[25] J. Kurzweil, Ordinary differential equations, Elsevier, Amsterdam, 1986.

[26] J. T. Londergan, J. P. Carini and D. P. Murdock, Binding and scattering in twodimensional systems, LNP, vol. m60, Springer, Berlin, 1999.

[27] E. Mourre, Absence of singular continuous spectrum for certain self-adjoint operators, Commun. Math. Phys. 78 (1981), 391-408.

[28] M. Reed and B. Simon, Methods of modern mathematical physics, III. Scattering theory, Academic Press, New York, 1979.

[29] _ Methods of modern mathematical physics, IV. Analysis of operators, Academic Press, New York, 1978.

[30] W. Renger and W. Bulla, Existence of bound states in quantum waveguides under weak conditions, Lett. Math. Phys. 35 (1995), 1-12.

[31] C. Y. H. Tsao and W. A. Gambling, Curvilinear optical fibre waveguide: characterization of bound modes and radiative field, Proc. R. Soc. Lond. A 425 (1989), $1-16$. 